\title{
Multi-Stage Bunch Compressors for the International Linear Collider *
}

\author{
P. Tenenbaum ${ }^{\dagger}$, T.O. Raubenheimer, SLAC, Stanford CA, USA \\ A. Wolski, LBNL, Berkeley, CA USA
}

\begin{abstract}
We present bunch compressor designs for the International Linear Collider (ILC) which achieve a reduction in RMS bunch length from $6 \mathrm{~mm}$ to $0.3 \mathrm{~mm}$ via multiple stages of compression, with stages of acceleration inserted between the stages of compression. The key advantage of multi-stage compression is that the maximum RMS energy spread is reduced to approximately $1 \%$, compared to over $3 \%$ for a single-stage design. Analytic and simulation studies of the multi-stage bunch compressors are presented, along with performance comparisons to a single-stage system. Parameters for extending the systems to a larger total compression factor are discussed.
\end{abstract}

\section{INTRODUCTION}

In order to meet its luminosity goals, the International Linear Collider (ILC) requires a value of $\beta_{y}^{*}$ between 150 $\mu \mathrm{m}$ and $600 \mu \mathrm{m}$, with $300 \mu \mathrm{m}$ being the nominal value [1]; because of the hourglass effect, this implies that the RMS bunch length at the collision point must also be on the order of $300 \mu \mathrm{m}$. Since single-bunch collective effects in the ILC damping ring will set a lower limit of approximately $6 \mathrm{~mm}$ on $\sigma_{z}$ in the damping ring, it will be necessary to compress the beam after extraction from the damping ring but prior to injection into the linac.

\section{Single Stage Compressor}

The TESLA TDR design incorporated a bunch compressor which reduced the RMS bunch length from $6 \mathrm{~mm}$ to 300 $\mu \mathrm{m}$ in a single stage of compression, with an RF section operating near its zero crossing followed by a wiggler [2] A version of the TDR compressor was developed which is appropriately reoptimized for the larger energy spread foreseen for the ILC damping ring compared to the TDR design $(0.15 \%$ versus $0.13 \%$ RMS $)$, with optics as shown in Figure 1. This compressor design has a number of unsatisfactory features, to wit:

- The final energy spread is extremely large, in excess of $3 \%$ RMS.

- Because the $T_{566}$ of the wiggler $\times$ the final energy spread is comparable to the final bunch length, it is necessary to compensate the $T_{566}$ by running the RF backphased rather than at the zero-crossing [3], which reduces the beam energy in the linac and increases the RMS energy spread at linac injection.

* Work supported by the US Department of Energy, Contracts DEAC02-76SF00515 and DE-AC03-76SF00098.

† quarkpt@slac.stanford.edu
- Even with $T_{566}$ compensation, the remaining nonlinearities in the compression process require that the bunch be slightly overcompressed, resulting in an even larger RMS energy spread.

- The compressor requires over $1200 \mathrm{MV}$ of RF cavities at a location where the bunch length is $6 \mathrm{~mm}$, resulting in potentially large emittance growth from offset or pitched RF cavities.

- Larger compression ratios are ruled out, since they would require even larger final energy spreads and more RF cavities at the long-bunch location.

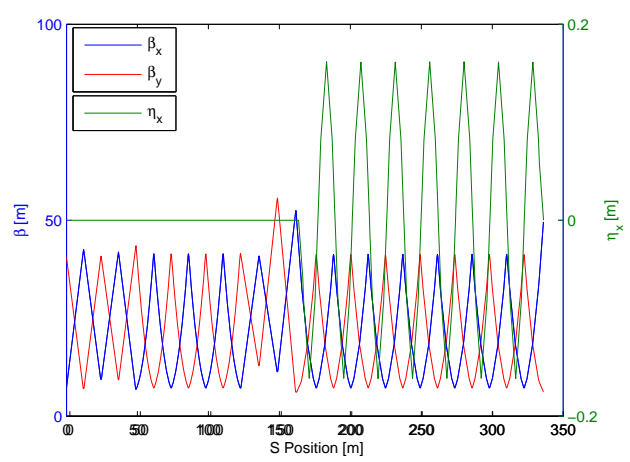

Figure 1: Twiss parameters of single-stage bunch compressor.

\section{MULTI-STAGE COMPRESSORS}

The drawbacks of the single-stage compressor can be mitigated by performing the bunch compression in multiple stages, with acceleration sections interleaved between the compressor stages. We consider a two-stage bunch compressor and a three-stage bunch compressor. In each configuration the initial compression is performed at the damping ring extraction energy of $5 \mathrm{GeV}$, and the second compression at $10 \mathrm{GeV}$.

\section{Two-Stage Bunch Compressor}

In the two-stage bunch compressor the bunch is initially compressed to $1.05 \mathrm{~mm}$ RMS length at $5 \mathrm{GeV}$, accelerated to approximately $10 \mathrm{GeV}$, and compressed to $300 \mu \mathrm{m}$ RMS length before injection into the main linac. Two variants of this compressor were considered: in the first variant ("A"), each stage performs a 90 degree rotation in the longitudinal phase plane; in the second variant ("B"), the initial stage of compression undercompresses the bunch, which 
reduces the total voltage needed in the second stage. Figure 2 shows the Twiss parameters of the "A" configuration (the " $\mathrm{B}$ " differs in detail but is qualitatively the same), and Table 1 shows the key parameters of the two variants.

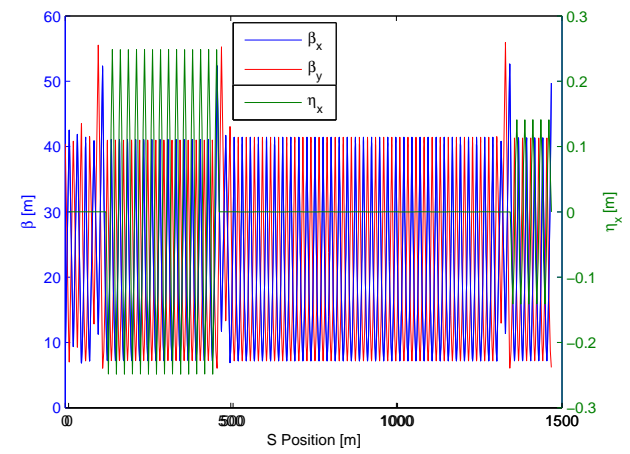

Figure 2: Twiss parameters of two-stage bunch compressor.

Table 1: Parameters of the two-stage bunch compressor.

\begin{tabular}{|l|c|c|c|c|}
\hline Parameter & \multicolumn{2}{|c|}{ Variant "A" } & \multicolumn{2}{c|}{ Variant "B" } \\
& Stage 1 & Stage 2 & Stage 1 & Stage 2 \\
\hline Voltage $[\mathrm{MV}]$ & 270 & 8600 & 610 & 5000 \\
Phase $\left[^{\circ}\right]$ & -100 & -40 & -110 & -25 \\
$\Delta \mathrm{E}[\mathrm{MeV}]$ & -46.9 & 6588 & -209 & 4532 \\
$R_{56}[\mathrm{~mm}]$ & -700 & -80 & -260 & -68 \\
$\sigma_{z}[\mu \mathrm{m}]$ & 1050 & 300 & 1050 & 300 \\
$\sigma_{\delta}[\%]$ & 0.9 & 1.3 & 1.9 & 1.5 \\
\hline
\end{tabular}

\section{Three Stage Bunch Compressor}

In the three stage bunch compressor, the bunch is initially compressed to an RMS length of $750 \mu \mathrm{m}$ via a $90^{\circ}$ phase space rotation. This is followed by a telescopic system, which uses 2 RF systems and 2 wigglers to reduce the RMS bunch length to $300 \mu \mathrm{m}$ while preserving the orientation of the beam ellipse in the longitudinal phase plane (note that despite the name, the three stage bunch compressor does not reduce the bunch length in each stage of longitudinal phase space manipulations). Figure 3 shows the Twiss parameters of this bunch compressor, and Table 2 its key parameters.

Table 2: Parameters of the three stage bunch compressor.

\begin{tabular}{|l|c|c|c|}
\hline Parameter & Stage 1 & Stage 2 & Stage 3 \\
\hline \hline Voltage $[\mathrm{MV}]$ & 380 & 3000 & 9700 \\
Phase $\left[{ }^{\circ}\right]$ & -98 & -80 & -65 \\
$\Delta \mathrm{E}[\mathrm{MeV}]$ & -53 & 521 & 4099 \\
$R_{56}[\mathrm{~mm}]$ & -500 & -78 & -53 \\
$\sigma_{z}[\mu \mathrm{m}]$ & 750 & 840 & 300 \\
$\sigma_{\delta}[\%]$ & 1.2 & 1.3 & 1.6 \\
\hline
\end{tabular}

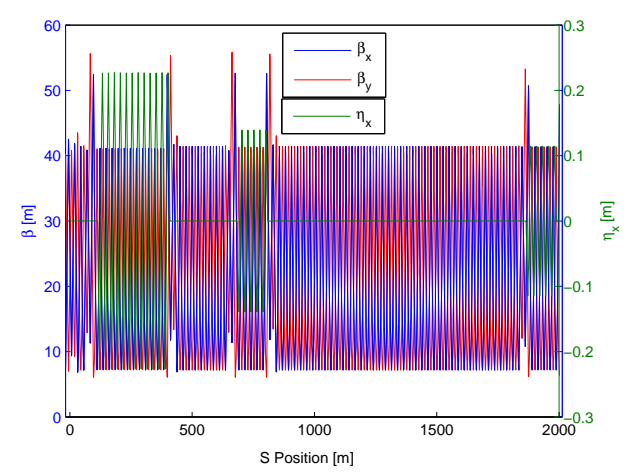

Figure 3: Twiss parameters of the three-stage bunch compressor.

\section{COMPARISON OF SYSTEM LENGTHS}

As shown in Figure 1, the total length of the singlestage bunch compressor is approximately 340 meters. This value does not accurately reflect the total lengthening of the ILC site required by the bunch compressor, since the single-stage model also decelerates the beam from $5 \mathrm{GeV}$ to $4.35 \mathrm{GeV}$; when the reacceleration to $5 \mathrm{GeV}$ is taken into account, the single-stage compressor adds 460 meters per side. Thus, the site must be lengthened by 920 meters to accommodate one single-stage bunch compressor for each beam.

The two stage bunch compressor adds a total of 2020 meters to the site for the "A" configuration or 1720 meters for the " $\mathrm{B}$ " configuration. The three stage configuration adds 3640 meters to the total site length. A portion of the additional length for the multi-stage compressors is required to preserve the option of compressing to a smaller final bunch length, which requires more RF voltage.

\section{LONGITUDINAL TOLERANCES}

Since the bunch compressors manipulate the longitudinal phase space of the ILC beam, errors in this system can lead to errors in the energy, energy spread, arrival time, or bunch length at the IP. In practice the most demanding tolerances are typically those which change the arrival time at the IP, since the beams must collide at the focal point in order to achieve maximum luminosity. Arrival time errors as large as $20 \%$ of $\beta_{y}^{*}$, or $80 \mu \mathrm{m}$, can cause noticeable luminosity reduction.

\section{Damping Ring Extraction Phase}

The two-stage "A" configuration bunch compressor is relatively sensitive to the extraction phase from the damping ring: a stable phase error in the damping ring comparable to the design bunch length of $6 \mathrm{~mm}$ leads to an arrivaltime error of almost $300 \mu \mathrm{m}$ at the IP. The "B" configuration is much less sensitive, with the same $6 \mathrm{~mm}$ extraction phase error leading to approximately $80 \mu \mathrm{m}$ IP arrival time; it is expected that a modest retuning could eliminate this 
sensitivity altogether. The three stage configuration is almost entirely insensitive to damping ring stable phase variations: a $6 \mathrm{~mm}$ error in the damping ring becomes an error of about $10 \mu \mathrm{m}$ at the IP.

\section{RF Phase and Amplitude}

All 3 bunch compressor configurations studied have RF phase tolerances on the order of $0.1^{\circ}$, and amplitude tolerances of $0.3 \%$. It is important to note that these tolerances are for systematic errors over a very large number of RF cavities, which will be supported by multiple klystrons. We therefore expect the klystron RF tolerances will probably be closer to $0.3^{\circ}$ and $1 \%$. Furthermore, the bunch compressor can take advantage of the long fill time for the superconducting RF cavities (approximately $0.5 \mathrm{msec}$ ) to measure and correct the amplitude and phase of the klystron RF prior to the arrival of the beam. Studies at the TESLA Test Facility have suggested that klystron phase and amplitude tolerances of $0.1^{\circ}$ and $0.1 \%$, respectively, can be achieved through such feedback techniques [4].

\section{TRANSVERSE SENSITIVITIES}

One of the most serious deficiencies of the single-stage bunch compressor is that it introduces a large energy spread at the injection point of the main linac, which leads to large emittance growth from misalignments. In order to study the sensitivities of the candidate bunch compressor systems in a comparative way, each compressor was combined with a linac which accelerates the beam to $250 \mathrm{GeV}$. Each combined system (compressor + linac) was then misaligned and steered (via 1:1 steering) to zero its BPM readings. Three sets of misalignments were studied:

- BPM offsets of $10 \mu \mathrm{m}$ RMS, all other components perfectly aligned

- RF structure offsets of $500 \mu \mathrm{m}$ RMS, all other components perfectly aligned

- RF structure pitch angles of $75 \mu \mathrm{m}$ RMS, all other components perfectly aligned.

For each class of misalignments, 100 randomly misaligned accelerators were generated and steered. The mean emittance dilution for each class would allow the relative sensitivity to dispersion, wakefields, and head-tail RF deflections ("crabbing") to be observed. In all cases, a beam with initial normalized vertical emittance of $20 \mathrm{~nm}$ was used; this is the nominal extracted emittance from the ILC damping ring.

Table 3 shows the mean growth in the vertical normalized emittance for each class of misalignments, for each combination of bunch compressor and main linac. Note that these results are for comparison purposes only and are not meant to be suggestive of the actual ILC emittance preservation performance; determining the actual
Table 3: Normalized vertical emittance growth, in nanometers, for steered bunch compressor+linac combinations with errors as described in the text.

\begin{tabular}{|l||c|c|c|c|}
\hline Error & 1 Stage & \multicolumn{2}{|c|}{ 2 Stage } & 3 Stage \\
& & A & B & \\
\hline BPM Offsets & 15.4 & 6.9 & 10.7 & 9.3 \\
RF Offsets & 3.3 & 1.4 & 1.9 & 2.0 \\
RF Pitches & 5.5 & 0.8 & 1.8 & 1.6 \\
\hline
\end{tabular}

post-linac emittance requires a simulation effort which is beyond the scope of this paper.

Each multi-stage configuration studied was found to be significantly better than the single stage bunch compressor for all classes of misalignments. This is because, in addition to limiting the RMS energy spread, the multi-stage compressors limit the number of RF cavities through which the $6 \mathrm{~mm}$ uncompressed bunch must pass, which reduces both the wakefield and crabbing emittance terms.

\section{FURTHER REDUCTION IN BUNCH LENGTH}

Reducing the RMS bunch length requires that the RMS energy spread be increased; since the single-stage bunch compressor already generates over 3\% RMS energy spread achieving $300 \mu \mathrm{m}$ RMS bunch length, it is clear that another factor of 2 reduction cannot be achieved by this system. The multi-stage bunch compressors can achieve shorter final bunches. In this mode of operation longitudinal tolerances are comparable to tolerances for $300 \mu \mathrm{m}$ final bunch length, as are emittance growth figures for offset or pitched RF cavities. Emittance growth from dispersion will increase to about $20 \mathrm{~nm}$ for the two-stage "A" case and about $28 \mathrm{~nm}$ for the two-stage "B" and three-stage cases.

\section{CONCLUSIONS}

We have examined three candidate multi-stage bunch compressors for the International Linear Collider. Each of the candidates has performance equal to or better than a single stage compressor, and each of the candidate designs can achieve final bunch lengths as small as $150 \mu \mathrm{m}$ RMS. The multi-stage compressors are somewhat longer than the single-stage compressor and require additional RF voltage.

\section{REFERENCES}

[1] T.O. Raubenheimer, "Suggested ILC Beam Parameter Range," (2005).

[2] TESLA Technical Design Report, Part II, Chapter 6 (2001).

[3] P. Emma, "Cost and Performance Optimization of the NLC Bunch Compressor," (1999).

[4] C. Adolphsen, private communication. 